\title{
Prevalence of Risk Factors of non-communicable Diseases in an Adult Population of Rural Bangladesh
}

\author{
Palash Chandra Banik¹, M Mostafa Zaman², Jasimuddin Ahmed², \\ Sohel Reza Choudhury ${ }^{3}$,Mohammad Moniruzzaman ${ }^{2}$ \\ ${ }^{1}$ Department of Noncommunicable Diseases, Bangladesh University of Health Sciences, Dhaka, \\ ${ }^{2}$ Division of NCD, Ekhlaspur Centre of Health (ECOH), ${ }^{3}$ National Heart Foundation Hospital \\ and Research Institute, Dhaka
}

Keywords:

Bangladesh, Cardiovascular

disease,

Noncommunicable

diseases,

Community

based survey

\begin{abstract}
:
Background:A community based survey was carried out to determine the distribution of major noncommunicable diseases risk factors as well as to explore the detection and treatment rate of diabetes mellitus and hypertension in rural adult population of Bangladesh.

Methods: The survey was done in 2006 among 545 individualsaged 25 to 64 years randomly drawn from a village (Ekhlaspur under Chandpur district) of Bangladesh. Information on diet, physical activity, alcohol and tobacco, and treatment history for hypertension and diabetes were collected. Height, weight, waist circumference, blood pressure, capillary blood glucose and total cholesterol were measured.

Results: There were 269 men and 276 women with the mean age of 43.9 years (standard deviation, 11.6 years). Six in ten (62.4\%) of them used tobacco in some form (smoking or smokeless), 93.7\% did not consume adequate fruits and vegetables (five servings or more), and 7.4\% of them had low physical activity. More than $6 \%$ were overweight or obese $\left(B M I \geq 25 \mathrm{~kg} / \mathrm{m}^{2}\right)$ and $10 \%$ had hypertension (blood pressure $\geq 140$ / $90 \mathrm{mmHg}$ or medication). Capillary blood measurement and history of medication combined provided a prevalence of $3.1 \%$ for diabetes while prevalence of hypercholesterolemia $(\geq 240 \mathrm{mg} / \mathrm{dL})$ was $2.9 \%$ based on capillary blood cholesterol level alone. Less than half of hypertensive and less than one-tenth diabetes subjects were receiving treatmentfor their respective conditions.

Conclusions: Prevalence of noncommunicable diseases risk factorsis fairly high even in thisruralBangladeshi sample. Treatment rate for hypertension and diabetes is quite low. Interventionsshould address lifestyle changes and treatment of diabetes and hypertension.
\end{abstract}

(Cardiovasc. j. 2018; 10(2): 126-134)

\section{Introduction:}

Non-communicable diseases (NCDs)cardiovascular diseases, cancer, diabetes, and chronic respiratory disease-in Bangladesh have already become major public health concern. ${ }^{1}$ Almost 60\% deaths, in Bangladesh, are due to NCDs. ${ }^{2}$ Both NCDs and their risk factors are showing an increasing trend..$^{3,4}$ These risk factors are tobacco use, low intake of fruits and vegetables, physical inactivity, obesity, excessive alcohol consumption, raised blood pressure, and raised blood glucose and cholesterol. ${ }^{5}$ Evidences show that the detection and treatment rate of diabetes mellitus and hypertension is also inadequate among Bangladeshi population. ${ }^{6,7} \mathrm{~A}$ few studies shown that NCD risk factors are also very common in Bangladeshi population in both rural and urban areas and there is tendency of clustering of risk factors. ${ }^{4,8,9}$ These risk factors control is less costly than treatment of the total NCDs. Therefore risk factor approach for NCD prevention has become successful in many countries. ${ }^{10}$ For any intervention to prevent and control of these NCDs, first of all we have to know the distribution of risk factors in the population. A standardized approach, popularly known as STEPS, ${ }^{11}$ is being promoted by the World Health Organization for knowing the risk factor distribution in population. The present study was designed following STEPS design to determine the NCD risk factors distribution as well as detection and treatment rates of

Address of correspondence:Palash Chandra Banik, Department of Noncommunicable Diseases, Bangladesh University of Health Sciences (BUHS), Dhaka, Bangladesh. E-mail: palashcbanik@gmail.com 
hypertension and diabetes mellitus in a rural Bangladeshi population in order to design an appropriate intervention for the community.

\section{Methods:}

STEPS survey in Bangladesh was done in 2006 in eligible adults (see below) as per WHO STEPS protocol with a partial adaptation of its questionnaire. ${ }^{11}$ It was done in three out of seven divisions of Bangladesh. This paper is further analysis of Ekhlaspur component of this survey, representing one of the three divisions, reported elsewhere. ${ }^{12}$

\section{Study area:}

This study was conducted in Ekhlaspur village of Matlab North upazila (sub-district) of the Chandpur district, where the Ekhlaspur Center of Health (ECOH) has started a tobacco control intervention. Ekhlaspur is an agricultural area and most adults of the village endure high level of physical activity because of traditional lifestyle. Area background has already been described in details elsewhere. ${ }^{13}$

\section{Sample selection:}

STEPS 2006 survey targeted for a total of 3000 subjects aged 25 to 64 years and $\mathrm{ECOH}$ was allocated 600 (300 men and 300 women). ${ }^{12}$ As of 2005 Ekhlaspur had 1088 households, mapping and listing of households were already available for an ongoing intervention. Required number of households (600) was identified, for this survey, in sequential order starting from the first one. The survey team included one physician and two enumerators from $\mathrm{ECOH}$. They underwent 3-day training before starting the field work. Data collection was done in the first quarter of 2006. A roster of eligible individuals was prepared and one individual per household was recruited using Kish table. ${ }^{14}$ Sample substitution was not allowed and no repeat call was done. Methods for ascertainment of key variables are given below:

Inquired (step 1) variables: All the core questions from STEPS questionnaire were used. Household information was adapted to local situation such as dwelling house's roof materials. The individual component included questions on tobacco (both smoking and smokeless), physical activity, alcohol as well as fruits and vegetable intake. Information was also obtained on treatment rate of hypertension and diabetes. Time spent on moderate and vigorous physical activities (during work, leisure time and commutation) were transformed into minutes per week. Physical activity for less than 150 minutes was considered low. ${ }^{15}$ Alcohol consumption information during lifetime, past 12 months and past 30 days was asked. Serving sizes of fruits and vegetables in a typical week were determined using show-cards or measuring cups (in case of cooked items). Information on drug treatment of hypertension and diabetes was sought and prescriptions (or medicine strips) were checked if necessary.

Measured (step 2) variables: Physical measurements were done for height, weight and blood pressure. Shoes and heavy clothing were removed before taking such body measurements. Blood pressure was measured using ordinary aneroid sphygmomanometers on the left arm while the participants were in sitting position after having a rest for about 5 min. Korotkoff phase $\mathrm{V}$ was taken as diastolic blood pressure. Mean of two readings taken two minutes apart was used in the statistical analysis.

Biochemical (step 3) variables: WHO STEPS recommended equipment. ${ }^{16}$ Acutrend GC, was used to determine glucose and cholesterol by finger pricking without much pressing the fingers. The subjects were on overnight fasting status (8-12 h). Diabetes was defined as having blood glucose level e"126 mg/dL or current medication for diabetes and high cholesterol was defined as e"240 mg/dL of cholesterol. ${ }^{16}$

Statistical analysis: Descriptive statistics like mean, median, standard deviation, inter quartile range were calculated for continuous variables. Student's t test was used, to compare mean levels in men and women. Prevalence of categorical variables was compared using Chisquare test. 95\% Confidence Interval estimation was also done. ${ }^{17}$ Standardization for the age distribution of the new WHO world standard population (2000-2025) ${ }^{18}$ was used to standardize the prevalence. All analyses were carried out using SPSS 20.0 version. 


\section{Results:}

A total of 545 (men 269, women 276) villagers out of 600 invitees participated (response rate: overall 91\%; men, 90\%; women, 92\%). The respondents had a 44 years mean age and 6 years of schooling, and more than three-quarter of them were married. Ninety five percent women were home makers whereas half of men were either agriculture laborers or farmer. Ninety eight percent respondents' houses were made of tin sheet. These socioeconomic backgrounds (Table I) are typical in rural Bangladesh in current days. ${ }^{19}$

Distribution and prevalence of risk factors:

Fruits and vegetables: Mean per capita consumption of fruits and vegetables was 2.7 servings per day (Table II). Majority (93.7\%) of the respondents was found not to consume the recommended amount of fruits and vegetables (minimum 5 servings/ day) (Table III).

Alcohol: Almost ninety seven per cent respondents were lifetime abstainer of alcohol. Only $1.3 \%$ respondents found drink alcohol, even a single sip,in past twelve months. More men (4.4\%) found to drink compared to women (0.4\%) during last 12 months (Table III).

Physical activity: The median time spent in doing vigorous and/or moderate physical activity was 380 minutes per week (men: 870, women: 300) (Table II). Of them $7.4 \%$ (men $9.2 \%$ and women $5.8 \%)$ had low level of physical activity $(<150$ minutes in a week)(Table III).

Tobacco use: The overall age standardized prevalence of current smoking and smokeless

Table-I

Sociodemographic and economic characteristics of the respondents.

\begin{tabular}{|c|c|c|c|c|c|}
\hline Variables & Category & $\begin{array}{l}\text { and women } \\
\mathrm{n}=545\end{array}$ & $\begin{array}{c}\text { Men } \\
\mathrm{n}=269\end{array}$ & $\begin{array}{l}\text { Women } \\
\mathrm{n}=276\end{array}$ & $p$-values \\
\hline \multicolumn{6}{|c|}{ Age in year } \\
\hline & $25-34$ & $17(21.5)$ & $63(23.4)$ & $54(19.6)$ & \multirow[t]{4}{*}{0.112} \\
\hline & $35-44$ & $38(25.3)$ & 77 (28.6) & $61(22.1)$ & \\
\hline & $45-54$ & $50(27.5)$ & 67 (24.9) & 83 (30.1) & \\
\hline & $55-64$ & $40(25.7)$ & $62(23.0)$ & $78(28.3)$ & \\
\hline \multicolumn{6}{|c|}{ Marital Status } \\
\hline & Unmarried & 3.9 & 6.3 & 1.4 & \multirow[t]{4}{*}{0.001} \\
\hline & Married & 85.5 & 93.7 & 77.5 & \\
\hline & Divorce and Separate & 1.5 & 0.0 & 2.9 & \\
\hline & Widow & 9.2 & 0.0 & 18.1 & \\
\hline \multicolumn{6}{|l|}{ Education } \\
\hline & No schooling & 35 & 29.4 & 40.6 & \multirow{4}{*}{0.001} \\
\hline & Any primary ( 1 to 5 years) & 41.7 & 40.5 & 42.8 & \\
\hline & Any secondary (6 to 10 years) & 19.6 & 24.5 & 14.9 & \\
\hline & Above secondary (11 years and above) & 3.7 & 5.6 & 1.8 & \\
\hline \multicolumn{6}{|c|}{ Roof of the house made of } \\
\hline & Cement, concrete or tiles & 1.7 & 1.1 & 2.2 & \multirow[t]{3}{*}{0.226} \\
\hline & Tin sheet & 98 & 98.1 & 97.8 & \\
\hline & Bamboo, thatches, straw, shacks etc. & 0.4 & 0.7 & 0.0 & \\
\hline \multicolumn{6}{|c|}{ Primary occupation } \\
\hline & Home maker & 48.3 & 0.4 & 94.9 & \multirow[t]{6}{*}{0.001} \\
\hline & Day laborer & 18.7 & 37.9 & 0.0 & \\
\hline & Farmer & 5.1 & 10 & 0.4 & \\
\hline & Business & 2.6 & 2.6 & 2.5 & \\
\hline & Unemployed & 13.9 & 27.1 & 1.1 & \\
\hline & Student and others $¥$ & 11.4 & 21.9 & 1.1 & \\
\hline
\end{tabular}

$¥$ retired, jobless (able), jobeless (unable) respondents are included in others. 
Table II

Distribution of the non-communicable diseases risk factors among the adult men and women aged 25-64 years in Eklaspur.

\begin{tabular}{|c|c|c|c|c|c|}
\hline Risk factors & Age (year) & Both sexes & Men & Women & $p$-values* \\
\hline \multicolumn{6}{|c|}{ Fruit/ vegetables, servings/day... } \\
\hline & $25-34$ & $3.0(1.4)$ & $3.3(1.2)$ & $2.5(1.5)$ & \\
\hline & $35-44$ & $2.7(1.0)$ & $2.8(1.0)$ & $2.6(1.1)$ & \\
\hline & $45-54$ & $2.6(1.3)$ & $2.9(1.4)$ & $2.3(1.1)$ & \\
\hline & $55-64$ & $2.6(1.5)$ & $2.9(1.6)$ & $2.2(1.3)$ & \\
\hline & Total & $2.7(1.3)$ & $3.0(1.3)$ & $2.4(1.2)$ & 0.001 \\
\hline
\end{tabular}

Physical activity, minute/week¥

$\begin{array}{cc}25-34 & 490(300-920) \\ 35-44 & 515(305-880) \\ 45-54 & 430(270-880) \\ 55-64 & 260(177.5-600) \\ \text { Total } & 380(260-880)\end{array}$

$\begin{array}{lll}890(760-1115) & 300(272-120) & \\ 880(750-1110) & 320(280-360) & \\ 880(750-1110) & 290(240-375) & \\ 560(140-877.5) & 300(180-300) & \\ 870(520-1100) & 300(240-360) & 0.001\end{array}$

Body mass index, $\mathrm{kg} / \mathrm{m}^{\wedge} 2 \ldots \ldots \ldots$

$25-34$
$35-44$
$45-54$
$55-64$
Total

$$
\begin{aligned}
& 20.3(2.4) \\
& 20.4(3.1) \\
& 20.7(2.8) \\
& 20.0(2.7) \\
& 20.4(2.8)
\end{aligned}
$$

$20.4(2.5)$
$20.1(2.8)$
$20.9(2.3)$
$21.6(2.4)$
$20.7(2.6)$

Systolic blood pressure, $\mathrm{mmHg} .$.

$$
\begin{aligned}
& 25-34 \\
& 35-44 \\
& 45-54 \\
& 55-64 \\
& \text { Total }
\end{aligned}
$$

Diastolic blood pressure, $\mathrm{mmHg} .$.

$$
\begin{aligned}
& 25-34 \\
& 35-44 \\
& 45-54 \\
& 55-64 \\
& \text { Total }
\end{aligned}
$$

Glucose, mg/dL...........

$$
\begin{aligned}
& 25-34 \\
& 35-44 \\
& 45-54 \\
& 55-64 \\
& \text { Total }
\end{aligned}
$$

Cholesterol, mg/dL...

\begin{tabular}{lllll}
$25-34$ & $170.1(19.0)$ & $167.3(21.4)$ & $173.2(15.8)$ & \\
$35-44$ & $177.9(26.1)$ & $172.3(24.1)$ & $186.7(26.9)$ & \\
$45-54$ & $179.5(27.0)$ & $180.5(33.6)$ & $178.7(20.8)$ & \\
$55-64$ & $183.0(30.2)$ & $177.1(32.5)$ & $188.3(27.8)$ & \\
\hline Total & $177.8(26.2)$ & $173.8(27.7)$ & $181.9(24.0)$ & 0.01 \\
\hline
\end{tabular}

...Mean (standard deviation); $¥$ Median (Inter quartile range written as, lower-upper), To convert cholesterol and glucose values to $\mathrm{mmol} / \mathrm{L}$, multiply by 0.02586 and 0.05551 , respectively

*Independent samples t-test was done between men compare to women 


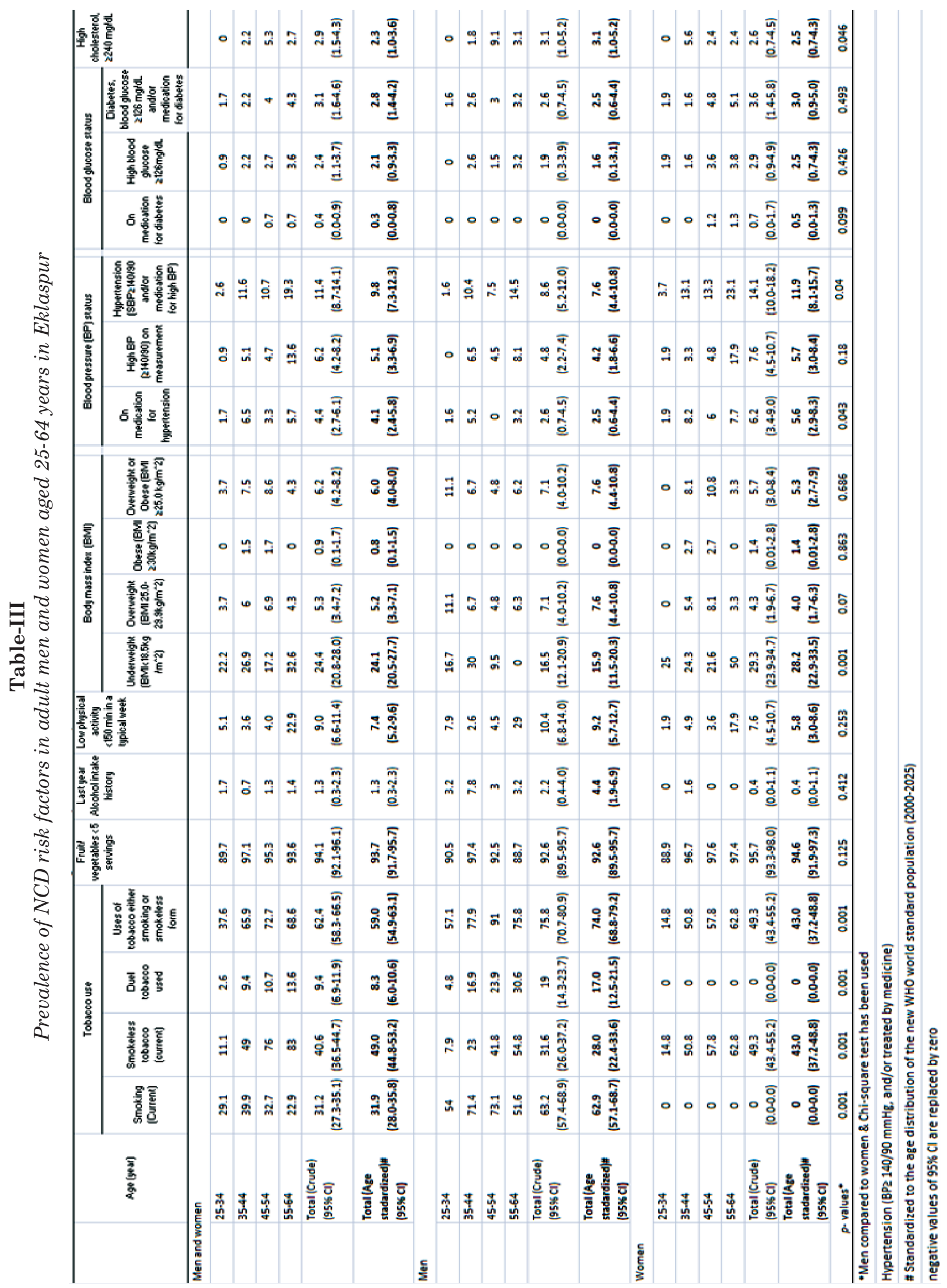


tobacco use was $31.9 \%$ and $49.0 \%$ respectively. Women did not smoke but used smokeless tobacco (43.0\%) more than men did (28.0\%).Among men, the prevalence of dual, and either form of tobacco use was $17.0 \%$ and $74.0 \%$ respectively (Table III).

Body mass index: The mean body mass index (BMI calculated as $\mathrm{Kg} /$ meter square) of the respondents was $20.4 \mathrm{~kg} / \mathrm{m}^{2}$, no significant difference by sex was found, $p=0.23$ (Table II). The overall prevalence of overweight or obese was $6.0 \%$ (men $7.5 \%$, women $5.3 \% ; p=0.69$ ). On the other hand, one quarter $(24.1 \%)$ of the respondents were underweight which was significantly higher $(p=0.001)$ in women $(28.2 \%)$ compared to men (15.9\%) (Table III).

Blood pressure status: The mean systolic, and diastolic blood pressure of the respondents was $114 \mathrm{mmHg}$ and $73 \mathrm{mmHg}$ respectively and it was fairly distributed in men and women (Table II). The overall age standardized prevalence of hypertension was $9.8 \%$ (Table III). It was significantly higher in women (11.9\%) than men $(7.6 \%)$. Of the total hypertensive respondents only $42 \%$ were receiving medication for hypertension.

Blood glucose status: The mean blood glucose of the respondents was $74.8 \mathrm{mg} / \mathrm{dL}$ (men 76.7 , women 73.2) (Table II). The overall age standardized prevalence of diabetes was $2.8 \%$. Of the total diabetic respondents, only $11 \%$ received medication as shown in Table III.

Blood Cholesterol status: The mean blood cholesterol of the respondents was $177.8 \mathrm{mg} / \mathrm{dL}$. It was significantly higher in women $(181.9 \mathrm{mg} /$ $\mathrm{dL}$ ) than men (173.8 mg/dL) (Table II). The overall age standardized prevalence of hypercholesterolemia was $2.9 \%$. The prevalence was a little higher in men (3.1\%) than women (2.6\%) (Table III). We did not seek any data on treatment for high cholesterol levels.

\section{Discussion:}

The present study is the first community based survey on NCD risk factors in rural Bangladesh, which covered variables of all three steps of STEPS survey. This study reports that all NCDs risk factors are highly prevalent among the villagers irrespective of their age and sex. In this study we have also observed a low detection as well as the treatment rate of hypertension and diabetes mellitus.

Fruit and vegetables:The present study observed very low fruits and vegetables intake, were similar findings also observed by other studies conducted in Bangladesh. ${ }^{4}$, ${ }^{9}$ Before conductionof this study, we had a perception that fruits and vegetables intakes in villagers will be high, because meat, fish and other nutritious foods are beyond their purchasing capacity due to the high cost, whereas, vegetables are comparatively inexpensive, cultivated locally and available in the market. But after this study, we found surprisingly inadequate fruit and vegetable intake among this population in spite of availability of seasonal fruits and plenty of vegetable. One of the possible reason could be people's ignorance about recommended daily intake (minimum 5 servings per day, 1 serving $=$ 80 gram).

Seasonal fruit, sometimes grown in plenty, are not popular because people do not consider them as good fruit and they are not very much aware about the nutritional value. In reality, these fruit are very nutritious and comparatively less costly than imported varieties. ${ }^{20}$ Therefore various awareness campaigns are very much needed to popularize local fruit. As proposed by Zaman MM etal. ${ }^{4}$ fruit should replace the existing 'misti' (sweets full of simple sugar and saturated fat) culture, and fruit should no more be considered as a sick person's diet but it should be eaten more frequently to prevent disease.

Alcohol: In context to the Bangladeshi culture and religion (in Islam alcohol is prohibited and $90 \%$ of Bangladeshi population are Muslim, ${ }^{21}$ alcohol sales and purchase in open market is forbidden. That's why, alcohol consumption among the Bangladeshi population is very low, and however in the present study we observed that $1.3 \%$ respondents were drinker and almost all of them were men which are higher than the national data. ${ }^{8}$ One of the possible causes of higher prevalence of alcohol consumption among this population could be due to misunderstanding regarding the question by the respondents, such as local 'energy' drinks might have been considered alcoholic drink. Further clarity on this is necessary. 
Physical activity: The global estimate for prevalence of physical inactivity among adults is $17 \%^{22}$ and in Bangladesh it varies from $34.5 \% 8$ to $38.6 \%{ }^{4}$. Our observed rate $(7.4 \%)$ is one-fifth of these rates. In our sample, one-quarter $(24.4 \%)$ of respondents were rather thin (BMI $<18.5 \mathrm{Kg} /$ $\mathrm{m}^{2}$ ).Most physical activities in our sample were from work related, household chores or otherwise. Leisure time physical activity is not popular at all. Commutation by bicycles and outdoor games could be encouraged. In the present study women were found have more physical activity than men as opposed to other studies. ${ }^{9}$ Additionally people in Ekhlaspur are more active than other areas of Bangladesh. ${ }^{9}$ Probably people, irrespective of sex, here are more actively engaged in farming, walking is still the primary mode of commutation. A substantial proportion of women participate in agricultural works including management of seasonal harvests.

Tobacco: Prevalence of both smoking (cigarette, bidi, etc.) and smokeless tobacco (zarda, gul, panmasala, etc.) use is high in men. We observed higher prevalence than other national level studies in spite of an ongoing anti-tobacco campaign, 8,23 Many men use tobacco in dual forms, smoking and smokeless. They are resistant to interventions and do not quit in spite of repeated counseling. Men's higher consumption, compared to women, of tobacco might be related to their economic independence and purchasing capacity.

Body mass index: Based on BMI almost one fourth of the respondents were underweight whereas similar findings have been observed in a national level representative study. ${ }^{9}$ Six percent respondents were overweight or obese which is one third of another national level report. ${ }^{8}$ A lower prevalence of obesity in Ekhlaspur might be due to higher level of physical activity of the people as stated above.

Hypertension: High blood pressure is a recognized intermediate risk factor in developing stroke and heart attacks. One meta-analysis of contemporary surveys published from 1995 to 2009 reported a prevalence of $13.5 \%$ whereas we report here a prevalence of $9.9 \%{ }^{24}$ This might be due to a higher level of physical activity and lower BMI of our subjects. In spite of being a common problem and simple to identify, hypertension detection and treatment status is far from adequate among the respondent which is actually reflecting the rules of halves theory for hypertension. ${ }^{25}$ The possible causes could be in most of the cases hypertension is asymptomatic and they do not have the knowledge about the possible complications of hypertension. Poverty could be one of the main reasons for less treatment of hypertension. Dietary salt must be targeted because its intake is very high (17 gm per day) in Bangladeshi people. $^{26}$

Diabetes: Although the prevalence we report here $(2.8 \%)$ is low, treatment rate is quite low. Higher level of physical activity could be responsible for a lower prevalence of diabetes in our sample. A $6.8 \%$ prevalence of diabetes has already been reported. ${ }^{27}$ There are lines of evidences that the prevalence of diabetes is rising in Bangladesh possibly because of recent substantial changes in lifestyle. ${ }^{4}$ On the other hands, the treatment history of diabetes patients by medication is very poor. The possible causes could be poverty and lack of awareness regarding diabetes complication.

Hypercholesterolemia: Although hypercholesterolemia is relatively low $(2.9 \%)$, borderline hypercholesterolemia is substantial(12.8\%). Zaman at el. ${ }^{12}$ reported in another article where the prevalence of hypercholesterolemia was $16 \%$. Such a striking difference between these two studies needs to be resolved. There is hardly any comparable data on cholesterol in Bangladeshi people. Future studies in Bangladeshi people should seek data on treatment of hypercholesterolemia as is done for hypertension and diabetes.

Finally the study findings cannot be generalized as it is conducted in a village population only. Lifestyle behaviors even in rural population can be different based on local environment and culture. However, this study is a snapshot of rural Bangladesh regarding NCD risk factors distribution which gives us an alarming picture. Risk reduction strategies have to be undertaken for primary prevention of NCDs. 


\section{Study Limitation}

Acutrend GC is WHO recommended equipment for measurement of glucose and cholesterol in STEPS surveys. However we do not know about its validity in our population. We cannot overrule a possibility of a bias for alcohol data. The study was conducted in a single location and results cannot be generalized to the whole rural population of Bangladesh.

\section{Conclusions}

This study gives us a snapshot of comprehensive NCDs risk factors profile of the villagers of Ekhlaspur. Prevalence of NCD risk factors is fairly high and both behavioral and biochemical risk factors are observed in age group and sex. On the basis of the findings different pragmatic control and prevention strategy could be developed by prioritizing the risk factors for the people with different age group and sex.

\section{Acknowledgements}

We are indebted to the then Director of Disease Control of the Directorate General of Health Services. We are grateful to the participants and local community leaders; without their help we could have achieved nothing. This study was part of STEPS 2006 and necessary fund was received from NCD Control Programme of the Directorate General of Health Services.

\section{Competing interest}

The authors declared that they have no competing interests.

\section{References:}

1. Global status report on noncommunicable diseases 2014. World Health Organization, Geneva, Switzerland, 2014. Available at http://www.who.int/nmh/publications/ncdstatus-report-2014/en/ [last accessed on 29 April, 2016]

2. Noncommunicable Diseases Country Profile 2014. World Health Organization, Geneva, Switzerland, 2014. Available athttp://www.who.int/nmh/publications/ncd-profiles-2014/ en/ [last accessed on May 12, 2016]

3. Zaman MM. Trends in non-communicable diseases (paper presented to the National Public Health Conference, 2013) Available at: http://www.iedcr.org/pdf/files/ NPHC\%20WEB/UPLOAD-4/Dr.Zaman.pdf [last accessed on 29 December 2013].

4. Zaman MM, Bhuiyan MR, Karim MN, MoniruzZaman, Rahman MM, Akanda AW, Fernando T. Clustering of noncommunicable diseases risk factors in Bangladeshi adults: An analysis of STEPS survey 2013. BMC Public Health
2015; 15:659. DOI 10.1186/s12889-015-1938-4

5. World Health Organization. The World Health Report 2002: Reducing Risks, Promoting Healthy Life. Geneva: WHO; 2002. P.1-230. Available at http://www.who.int/whr/ 2002/en/whr02_en.pdf?ua=1 [last accessed on 1 May 2016]

6. Rahman MS, Akter S, Abe SK, Islam MR, Mondal MNI, Rahman JAMS, et al.. Awareness, Treatment, and Control of Diabetes in Bangladesh: A Nationwide Population-Based Study. PLoS ONE 2015; 10(2):e0118365. doi:10.1371/ journal. pone.0118365

7. Islam FMA, Bhuiyan A, Chakrabarti R, Rahman MA, Kanagasingam Y and Hiller JE. Undiagnosed hypertension in a rural district in Bangladesh: The Bangladesh Population-based Diabetes and Eye Study (BPDES). Journal of Human Hypertension 2016; 30:252-259.

8. Zaman MM, Rahman M, Rahman M, Bhuiyan MR, Karim M, Chowdhury MJ. Prevalence of risk factors for noncommunicable diseases in Bangladesh: Results from STEPS survey 2010. Indian J Public Health 2016;60: $17-25$.

9. Moniruzzaman M, Zaman M, Islam MS, Ahasan HAMN, Kabir H, Yasmin R. Physical activity levels in Bangladeshi adults: results from STEPS survey 2010. Public Health 2016; http://dx.doi.org/10.1016/j.puhe.2016.02.028

10. Nikolic IA, Stanciole AE, Zaydman M. 2011. Chronic emergency: why NCDs matter. Health, Nutrition and Population (HNP) discussion paper. Washington, DC: World Bank. http://documents.worldbank.org/curated/en/ 2011/07/14867595/chronic-emergency-ncds-matter

11. Bonita R, deCourten M, Dwyer T, Jamrozik K, Winkelmann R. Surveillance of risk factors for noncommunicable diseases: The WHO STEPwise approach. Geneva, Switzerland: World Health Organization; 2002.

12. Zaman MM, Choudhury SR, Ahmed J, Talukder MH, Rahman AHMS. Blood glucose and cholesterol levels in adult population of Bangladesh: Results from STEPS 2006 survey. Indian Heart Journal 2016;64:52-56. http:// dx.doi.org/10.1016/j.ihj.2015.06.031

13. Zaman MM, Choudhury SR, Ahmed J, Hussain SM, Sobhan SM, Turin TC. Prevalence of Stroke in a Rural Population of Bangladesh. Glob Heart 2015; 10(4):333-4. doi: 10.1016/j.gheart.2014.04.007.

14. Kish L. A procedure for objective respondent selection with the household. J Am Stat Assoc 1949;44:380-387.

15. American Heart Association. Recommendations for Physical Activity in Adults. Available at http:// www.heart.org/HEARTORG/HealthyLiving/ PhysicalActivity/FitnessBasics/American-HeartAssociation-Recommendations-for-Physical-Activity-inAdults_UCM_307976_Article.jsp\#.VtI1_c5OK00 [last accesses on 28 February 2016]

16. World Health Organization. STEPwise approach to surveillance (STEPS). Available at http://www.who.int/chp/ steps/Part4_Section4.pdf [last accessed on 28 February 2016]. 
17. Lisa Sullivan. Confidence Intervals. Boston University School of Public Health. Available at http:// sphweb.bumc.bu.edu/otlt/MPH-Modules/BS/ BS704_Confidence_Intervals/BS704_Confidence_ Intervals print.html (accessed on 12 June 2016)

18. Ahmad OB, Boschi-Pinto C, Lopez AD Murray CJL, Lozano R, Inoue M. Age standardization of rates: a new who standard. GPE Discussion Paper Series: No.31. EIP/ GPE/EBD. World Health Organization 2001.

19. National Institute of Population Research and Training, Ministry of Health and Family Welfare. Bangladesh Demographic and Health Survey 2014. Dhaka, Bangladesh.

20. S Jahan, Gosh T, Begum M, Saha BK. Nutritional Profile of Some Tropical Fruits in Bangladesh: Specially AntiOxidant Vitamins and Minerals. Bangladesh Journal of Medical Science 2011;10(2):95-103.

21. Bangladesh Buruae of Educational Information and Statistics". Available at Banbeis.gov.bd. Retrieved 28 May 2016.

22. Mathers CD, Loncar D. Projection of global mortality and burden of disease from 2002 to 2030. PLoS Med. 2006;3:442
23. World Health Organization. Global Adult Tobacco Survey: Factsheet Bangladesh 2009. Country office for Bangladesh, WHO 2009. Available at http://www.who.int/tobacco/ surveillance/fact_sheet_of_gats_bangladesh_2009.pdf (accessed on 29 May 2016)

24. Moniruzzaman, Taleb A, Rahman S, Acharyya A, Islam FA, Ahmed MSA, et al. Prevalence of hypertension among the Bangladeshi adult population: a meta-analysis. Regional Health Forum. 2013;17:15-19.

25. Deepa R, Shanthirani CS, Pradeepa R, Mohan V. Is the 'rule of halves' in hypertension still valid?-Evidence from the Chennai Urban Population Study. J Assoc Physicians India 2003;51:153-157.

26. Zaman MM, Choudhury SR, Ahmed J, Khandker RK, Rouf MA, Malik A. Salt intake in an adult population of Bangladesh. Global Heart (In press: DOI 10.1016/ j.gheart.2016.05.005)

27. Rahim MA, Akhtar H, Khan AKA, Sayeed MA, Ali SMK, Vaaler S. Rising prevalence of type 2 diabetes in rural Bangladesh: A population based study. Diabetes Res Clin Pract 2007;77:300-305. 